\title{
The RoboCup Nanogram League: An Opportunity for Project-Based Undergraduate Education in Microsystems
}

\author{
Samara L. Firebaugh \\ United States Naval Academy \\ firebaug@usna.edu
}

\author{
Jenelle Armstrong Piepmeier \\ United States Naval Academy \\ piepmeie@usna.edu
}

\begin{abstract}
The new "nanogram league" of the international RoboCup competition creates an opportunity for project-based, undergraduate, microsystems education. The RoboCup problem was broken into three parts: general microrobot design and fabrication, machine vision and control, and design of a microrobot system to perform the RoboCup tasks. The design and construction of microrobots were the focus for a senior elective on microelectromechanical systems (MEMS). Concurrently, the vision portions of the problem were used as a project for a Machine Vision course. Students from both classes then formed design teams to create a system for competition.
\end{abstract}

\section{Introduction}

An undergraduate microelectromechanical systems (MEMS) course presents unique challenges. MEMS require knowledge of many disparate subjects. How does one form a course with cohesion and depth?

A project-based approach provides the necessary framework. In project-based learning, students work in groups to solve a challenging problem [1]. Projectbased learning has been applied in several undergraduate institutions, where it was found to increase student interest and motivation [2-4].

The challenge for the educator when formulating a project-based course is to determine a suitable problem. For a course on MEMS at the United States Naval Academy, we used a robotics competition, RoboCup [5]. This competition creates many interdisciplinary educational opportunities in MEMS as well as vision-based robotic control.

\section{The Problem}

The demonstration competition to be held at the Georgia Institute of Technology in July 2007 will consist of three compulsory events: a 2 millimeter dash, a slalom drill, and a ball-handling drill. In addition, teams can participate in the RoboCup Dance event. All events are viewed through a microscope.

The playing field has a layer of interdigitated electrodes, coated by a thin insulating layer. Defenders consist of photoresist patterned at various points on the field of play, and thin-film discs of silicon nitride serve as balls. Each player must operate without any physically connected tethers. The robots are controlled by a voltage waveform applied to the underlying electrode array on the playing field.

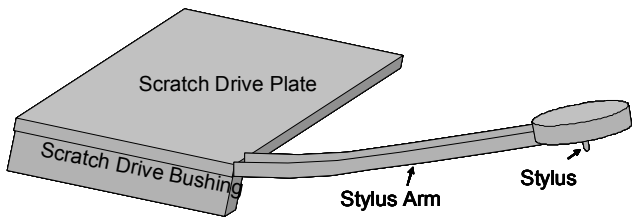

Figure 1. Illustration of microrobot

The inspiration for the competition, illustrated in Fig. 1, is a microrobot built by Donald et al. at Dartmouth [6]. The Dartmouth microrobots are $60 \mu \mathrm{m}$ by $250 \mu \mathrm{m}$ by $10 \mu \mathrm{m}$. Forward motion is accomplished via scratch drive actuation, described in [6]. Turning is accomplished by bringing the arm down in contact with the substrate while stepping the robot forward, so that the robot moves in an arc about the stylus.

The Dartmouth microrobots were built using a surface micromachining process that combined a commercial fabrication process (PolyMUMPS [7]) with a handful of additional process steps in order to deposit a thin layer of tensile material on the turning arm. This step is necessary so that the scratch drive actuation voltages are nested within the pull-in and release voltages from the stylus.

The task set before our students was to study and reproduce the Dartmouth robots, and then to design new robots for the RoboCup competition. This problem required investigation of processing techniques, material properties, electrostatic actuation, machine vision and control methods. The combination of commercial and student processing allowed for hands-on exposure to microfabrication while also 
introducing them to layout tools and the application of design rules in a commercial process.

\section{The MEMS Course}

In the MEMS course, the students began by reading the RoboCup problem statement and technical papers on the Dartmouth microrobots $[6,8]$. The students then determined the topics they would need to study and set an aggressive schedule.

After reviewing fabrication techniques, they derived the design equations for the scratch drive actuator and the pull-in and release voltages for the stylus arm. They learned the layout software and designed structures for the PolyMUMPS run. While the students awaited the return of their devices, they trained on the equipment in the campus microfabrication facility and developed recipes for lithography and metal evaporation. A few implementation problems arose; however, by the end of the semester the students demonstrated forward motion with tethered microrobots.

\section{Machine Vision}

A path planning and feedback control scheme was necessary to control the microrobots, which are handicapped to forward motion and left turns. The block diagram for a visually servoed microrobot system is shown in Fig. 2.

A Machine Vision course in the Systems Engineering Department addressed the visual feedback problem. This is a project-based course, for which the microrobotics task was only one of many projects. Using a captured video sequence of the Dartmouth microrobot, students prototyped image processing algorithms. RoboCup also provided opportunities for application-related system integration discussions such as camera selection and processing speeds.

\section{Senior Capstone Project}

At the end of the first semester, the students from the MEMS course were joined by a student from the Machine Vision course and two new students to form senior design project teams. Each team was made up of a combination of experienced and new students, with an experienced student serving as team leader. These teams created microrobots specific to the soccer agility tasks, and combined these robots with machine vision and control. The teams will compete against each other at the conclusion of the spring semester, and then combine to take the winning system to competition in Atlanta in July.

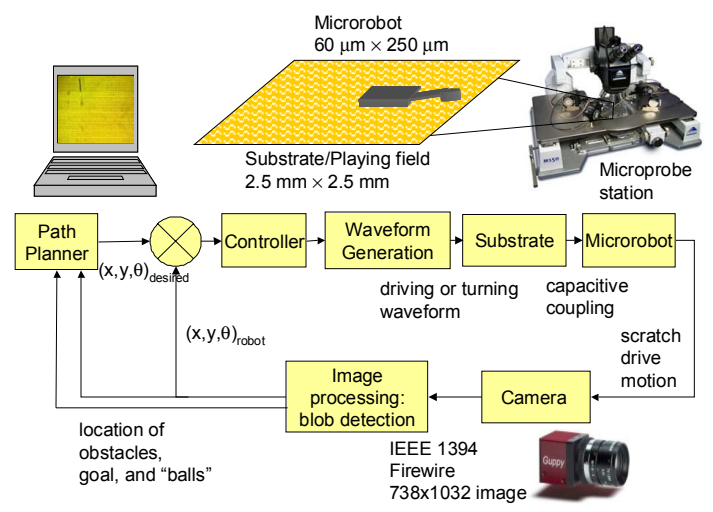

Figure 2. Block diagram of control system

\section{Summary}

The students in the MEMS course and machine vision course were evaluated by lab reports, which indicated a mastery of the greater course learning objectives. In course evaluations, the students specifically praised the problem-based structure of the courses. They were also elated by their success with the tethered microrobots and enthusiastic about continuing the project into the spring semester. The senior design project will be evaluated by technical reports and the final design presentation. Whether or not the team succeeds in Atlanta, the RoboCup problem has provided an excellent undergraduate microsystems education experience.

\section{References}

[1] Gwen Solomon, "Project-Based Learning: A Primer," Technology \& Learning, vol. 23, pp. 20-30, 2003.

[2] Mark Somerville et al., "The Olin Curriculum: Thinking Toward the Future," IEEE Transactions on Education, vol. 48, pp. 198-205, 2005.

[3] Walter Daems et al., "PeopleMover: An Example of Interdisciplinary Project-Based Education in Electrical Engineering," IEEE Transactions on Education, vol. 46, pp. 157-167, 2003.

[4] Javier Macias-Guarasa et al., "A Project-Based Learning Approach to Design Electronic Systems Curricula," IEEE Transactions on Education, vol. 49, pp. 389-397, 2006.

[5] "Robocup Web Site," 24 January 2007, $<\mathrm{http} / / /$ www.robocup.org/>.

[6] Bruce R. Donald et al., "An Untethered, Electrostatic, Globally Controllable MEMS Micro-Robot," Journal of Microelectromechanical Systems, vol. 15, pp. 1-15, 2006.

[7] MEMSCAP, Inc., www.memscap.com.

[8] Bruce R. Donald et al., "Power Delivery and Locomotion of Untethered Microactuators," Journal of Microelectromechanical Systems, vol. 12, pp. 947-959, 2003. 